Tohoku Math. J.

65 (2013), 523-543

\title{
SECOND VARIATIONAL FORMULA AND THE STABILITY OF LEGENDRIAN MINIMAL SUBMANIFOLDS IN SASAKIAN MANIFOLDS
}

\author{
TORU KAJIGAYA
}

(Received August 22, 2012, revised February 6, 2013)

\begin{abstract}
In this paper, we investigate compact Legendrian submanifolds $L$ in Sasakian manifolds $M$, which have extremal volume under Legendrian deformations. We call such a submanifold L-minimal Legendrian submanifold. We derive the second variational formula for the volume of $L$ under Legendrian deformations in $M$. Applying this formula, we investigate the stability of L-minimal Legendrian curves in Sasakian space forms, and show the L-instability of L-minimal Legendrian submanifolds in $S^{2 n+1}(1)$. Moreover, we give a construction of L-minimal Legendrian submanifolds in $\boldsymbol{R}^{2 n+1}(-3)$.
\end{abstract}

1. Introduction. In [10], [11], Y. G. Oh introduced the notion of Hamiltonianminimal (H-minimal) Lagrangian submanifolds in Kähler manifolds. Such a submanifold is a critical point of the volume functional under the Hamiltonian deformation. This is an extension of the notion of minimal submanifold, and has been studied by many authors (for example, [7], [8], [11], [14] and see references therein). An H-minimal Lagrangian submanifold is called Hamiltonian-stable (H-stable) if the second variation is non-negative for any Hamiltonian deformation. Oh studied H-stablity of some examples of H-minimal Lagrangian submanifold in a specific Kähler manifold ([10], [11]). For example, the real projective space $\boldsymbol{R} \boldsymbol{P}^{n}$ and the Clifford torus in $\boldsymbol{C} P^{n}$, and the standard tori in $\boldsymbol{C}^{n}$ are H-stable. Besides these examples, Schoen and Wolfson studied the H-stablity of two-dimensional H-minimal Lagrangian cones ([14]), and Iriyeh studied the three-dimensional case ([8]). Furthermore, in [1], Amarzaya and Ohnita proved that all compact Lagrangian submanifolds with parallel second fundamental form in $\boldsymbol{C}^{n}$ and $\boldsymbol{C} P^{n}$ are H-stable.

On the other hand, there is a notion of Sasakian manifolds, which is an odd-dimensional counterpart to Kähler manifolds. In Sasakian manifolds, we consider Legendrian-minimal $(L$ minimal) Legendrian submanifolds which correspond to H-minimal Lagrangian manifolds in Kähler manifolds. An L-minimal Legendrian submanifold is a critical point of the volume function under the Legendrian deformation (for more details, see Section 3). In [7], [8], the authors constructed examples of L-minimal Legendrian submanifolds in odd-dimensional unit spheres (in [7], such submanifolds are called $C$-minimal). Moreover, they show that a certain L-minimal Legendrian submanifold in a Sasakian manifold is related to an H-minimal Lagrangian submanifold in a Kähler manifold. For example, the cone over a Legendrian

2010 Mathematics Subject Classification. Primary 53C42; Secondary, 53C25.

Key words and phrases. Legendrian submanifold, Sasakian manifold, Legendrian stability. 
submanifold in the unit sphere is an H-minimal Lagrangian submanifold in $\boldsymbol{C}^{n}$ if and only if its link is L-minimal ([7], [8]). Another correspondence is shown in the Hopf fibration $\pi: S^{2 n+1}(1) \rightarrow \boldsymbol{C} P^{n}$, that is, a Legendrian submanifold $L$ in $S^{2 n+1}(1)$ obtained as the lift of a Lagrangian submanifold $\bar{L}$ in $C P^{n}$ is L-minimal if and only if $\bar{L}$ is H-minimal.

It is natural to ask whether an L-minimal Legendrian submanifold is stable under Legendrian deformations. The case of minimal Legendrian submanifolds was studied in [12]. In the present paper, we derive the second variational formula for L-minimal Legendrian submanifolds in Sasakian manifolds, and study their stability.

THEOREM 1.1. Let $\iota: L^{n} \rightarrow M^{2 n+1}$ be a compact L-minimal Legendrian immersion into a Sasakian manifold $\left(M^{2 n+1}, \phi, \xi, \eta, g\right)$, and let $\left\{\iota_{t}\right\}_{-\varepsilon \leq t \leq \varepsilon}$ be a Legendrian deformation of $L^{n}$ with $\iota_{0}=\iota$, where we assume that the variational vector field $V=f \xi+V_{\mathcal{H}}$ is normal to $\iota$. When L has a boundary, we also assume that the deformation fixes the boundary. Then we have

$$
\begin{aligned}
\left.\frac{d^{2}}{d t^{2}}\right|_{t=0} \operatorname{Vol}\left(\iota_{t}(L)\right)= & \int_{L}\left\{\frac{1}{4}|\Delta f|^{2}-2\left|V_{\mathcal{H}}\right|^{2}-\overline{\operatorname{Ric}}\left(V_{\mathcal{H}}\right)\right. \\
& \left.-2 g\left(B\left(\phi V_{\mathcal{H}}, \phi V_{\mathcal{H}}\right), H\right)+g\left(V_{\mathcal{H}}, H\right)^{2}\right\} d v_{L},
\end{aligned}
$$

where $\Delta$ is the Laplace-Beltrami operator acting on $C^{\infty}(L), \overline{\operatorname{Ric}}$ is the Ricci tensor of $M^{2 n+1}$, $B$ is the second fundamental form of $\iota$, and $H$ is the mean curvature vector of $\iota$.

We call an L-minimal Legendrian submanifold Legendrian-stable (L-stable) if the second variation is non-negative for any Legendrian deformation. Applying Theorem 1.1, we firstly investigate the L-stability of L-minimal Legendrian curves in the Sasakian space forms $S^{3}(1), \boldsymbol{R}^{3}(-3)$ and $S L(2, \boldsymbol{R})$, and determine the L-stability of these curves. In particular, we show that there exist L-stable L-minimal Legendiran closed curves in $\operatorname{SL}(2, \boldsymbol{R})$.

Moreover, we apply Theorem 1.1 to investigate the L-stability of L-minimal Legendrian submanifolds in the odd-dimensional unit sphere $S^{2 n+1}(1)$. Recall that there are some Hstable H-minimal Lagrangian submanifolds in $\boldsymbol{C} P^{n}$. In contrast to this fact, we prove the following L-instability theorem:

THEOREM 1.2. All closed L-minimal Legendrian submanifolds in $S^{2 n+1}(1)$ are Lunstable.

In Section 2, we prepare notation, and review properties of Sasakian manifolds and Legendrian submanifolds. In Section 3, we define the notion of L-minimal Legendrian submanifolds. Then, in Section 4, we give a proof of Theorem 1.1. In the last section, we investigate the stability of some examples of L-minimal Legendrian submanifolds in Sasakian space forms, and give a proof of Theorem 1.2. We also discuss the existence of L-minimal Legendrian submanifolds in $\boldsymbol{R}^{2 n+1}(-3)$.

2. Preliminaries. A $(2 n+1)$-dimensional contact manifold $\left(M^{2 n+1}, \eta\right)$ is called a Sasakian manifold ([5]) if it admits a $(1,1)$-tensor $\phi$, a characteristic vector field $\xi$ and an 
associated metric $g$ satisfying

$$
\begin{aligned}
\phi^{2}= & -I d+\eta \otimes \xi, \quad \eta(\xi)=1, \quad \phi \xi=0, \quad \eta \circ \phi=0, \\
& g(\phi X, \phi Y)=g(X, Y)-\eta(X) \eta(Y), \quad \eta(X)=g(X, \xi), \quad d \eta(X, Y)=g(X, \phi Y), \\
& \left(\bar{\nabla}_{X} \phi\right) Y=g(X, Y) \xi-\eta(Y) X,
\end{aligned}
$$

for any vector fields $X, Y$ on $M$, where $\bar{\nabla}$ denotes the Levi-Civita connection with respect to $g$. The last condition is called the normal condition, which corresponds to the Kähler condition on Kähler manifolds. We note that an odd-dimensional Riemannian manifold $\left(M^{2 n+1}, g\right)$ admits a Sasakian structure if and only if the Riemannian cone $C(M)=M \times \boldsymbol{R}^{+}$over $M$ admits a Kähler structure ([6]).

Sasakian manifolds also satisfy

$$
\left(\bar{\nabla}_{X} \xi\right)=-\phi X
$$

and have the curvature properties

$$
\begin{gathered}
\bar{R}(X, \xi) \xi=X-\eta(X) \xi, \\
\bar{R}(X, Y) \xi=\eta(Y) X-\eta(X) Y, \\
\bar{R}(\xi, X) Y=\left(\bar{\nabla}_{X} \phi\right) Y, \\
g(\bar{R}(\phi X, \phi Y) \phi Z, \phi W)=g(\bar{R}(X, Y) Z, W), \quad \text { if } X, Y, Z, W \perp \xi,
\end{gathered}
$$

where $\bar{R}$ denotes the curvature tensor of $M$ with respect to $\bar{\nabla}$.

A Sasakian manifold $\left(M^{2 n+1}, \phi, \xi, \eta, g\right)$ is called $\eta$-Einstein if the Ricci tensor is given by

$$
\overline{\mathrm{Ric}}=a g+(2 n-a) \eta \otimes \eta
$$

for some function $a \in C^{\infty}(M)$. We remark that if $n>1$, then $a$ must be constant ([5]). A Sasakian manifold $M^{2 n+1}$ is called a Sasakian space form if it has constant $\phi$-sectional curvature $c$, i.e.,

$$
K\left(X_{p}, \phi X_{p}\right) \equiv c, \quad \text { for } X_{p} \in \xi^{\perp}, \quad p \in M,
$$

where $K\left(X_{p}, \phi X_{p}\right)$ is the sectional curvature of the horizontal subspace spanned by $\left\{X_{p}, \phi X_{p}\right\} \subset T_{p} M$. A Sasakian space form is also $\eta$-Einstein with $a=\{n(c+3)+c-1\} / 2$. We denote the Sasakian space form by $M^{2 n+1}(c)$. Basic examples of Sasakian space forms are the odd-dimensional Euclidean space $\boldsymbol{R}^{2 n+1}(-3)$, and the unit sphere $S^{2 n+1}(1)$ with the standard Sasakian structure ([5]).

Let $L^{n}$ be an $n$-dimensional compact manifold with or without boundary, and $\iota: L^{n} \rightarrow$ $M^{2 n+1}$ an isometric immersion. Then $L^{n}$ is called Legendrian if $\iota^{*} \eta=0$. This condition implies that $\iota\left(L^{n}\right)$ is an integral submanifold tangent to the distribution $\mathcal{H}:=\operatorname{Ker} \eta=\xi^{\perp}$ with the maximal dimension $n$. In the following, we may identify $L^{n}$ with $\iota\left(L^{n}\right)$. In [13], it is shown that a Legendrian immersion $\iota$ satisfies the following.

(i) Anti-invariant condition: $\iota_{*} T_{p} L \perp \phi\left(\iota_{*} T_{p} L\right)$ for any $p \in L$. 
(ii) The second fundamental form $B$ of $\iota$ takes values in Ker $\eta$.

The anti-invariant condition implies that the normal bundle $N L$ of $\iota$ has the orthogonal splitting $N_{\iota(p)} L=\boldsymbol{R} \xi_{\iota(p)} \oplus \phi\left(\iota_{*} T_{p} L\right)$ for any $p \in L$. Under this splitting, we decompose a normal vector field $V \in \Gamma(N L)$ into $V=f \xi+V_{\mathcal{H}}$, where $f=g(V, \xi)=\eta(V)$. Also, the anti-invariant condition implies that there is a natural identification between $\phi\left(\iota_{*} T_{p} L\right)$ and the dual space $T_{p}^{*} L$ of $T_{p} L$ given by

$$
\begin{aligned}
\phi\left(\iota_{*} T_{p} L\right) & \stackrel{\sim}{\rightarrow} T_{p}^{*} L \\
V_{p} & \left.\mapsto \alpha_{V p}:=-\frac{1}{2} \iota^{*}\left(V_{p}\right\rfloor d \eta_{p}\right)=\iota^{*}\left\{g\left(\cdot, \phi V_{p}\right)\right\} .
\end{aligned}
$$

Hence we have a natural isomorphism between $\Gamma(N L)$ and $C^{\infty}(L) \oplus \Omega^{1}(L)$ (see also Lemma 3.2 in [12]):

$$
\begin{aligned}
& \Phi: \Gamma(N L) \rightarrow C^{\infty}(L) \oplus \Omega^{1}(L), \\
& \Phi(V)=\left(\eta(V), \alpha_{V}\right) .
\end{aligned}
$$

Moreover, we have

$$
g(V, W)=\eta(V) \eta(W)+g^{*}\left(\alpha_{V}, \alpha_{W}\right)
$$

for any $V, W \in \Gamma(N L)$, where $\left.\alpha_{V}=-\frac{1}{2} \iota^{*}(V\rfloor d \eta\right)$ and $g^{*}$ is the metric on $\Omega^{1}(L)$ induced from $\iota^{*} g$.

In the following we omit $\iota$. We denote by $\nabla$ and $\nabla^{\perp}$ the connection induced from $\bar{\nabla}$ on $T L$ and $N L$, respectively, and denote by $\nabla$ the induced connection on $T^{*} L$.

LEMMA 2.1. For $V=f \xi+V_{\mathcal{H}} \in \Gamma(N L)$ and $X \in \Gamma(T L)$, we have

$$
\Phi\left(\nabla_{X}^{\perp} V\right)=\left(X f-\alpha_{V}(X), \nabla_{X} \alpha_{V}-f \alpha_{\phi X}\right) .
$$

Proof. By (2) and (7), the first component becomes

$$
\begin{aligned}
\eta\left(\nabla_{X}^{\perp} V\right) & =g\left(\nabla_{X}^{\perp} V, \xi\right)=g\left(\bar{\nabla}_{X} V, \xi\right) \\
& =\bar{\nabla}_{X} g(V, \xi)-g\left(V, \bar{\nabla}_{X} \xi\right)=X(\eta(V))-g(V,-\phi X) \\
& =X(\eta(V))-\alpha_{V}(X) .
\end{aligned}
$$

Next we calculate the second component. For any $Y \in \Gamma(T L)$, from (7) and the normal condition, we have

$$
\begin{aligned}
\alpha_{\nabla_{X}^{\perp} V}(Y) & =-g\left(\nabla_{X}^{\perp} V, \phi Y\right) \\
& =-g\left(\bar{\nabla}_{X} V, \phi Y\right) \\
& =-\bar{\nabla}_{X} g(V, \phi Y)+g\left(V, \bar{\nabla}_{X}(\phi Y)\right) \\
& =\nabla_{X}\left(\alpha_{V}(Y)\right)+g\left(V,\left(\bar{\nabla}_{X} \phi\right)(Y)+\phi\left(\bar{\nabla}_{X} Y\right)\right) \\
& =\left(\nabla_{X} \alpha_{V}\right)(Y)+\alpha_{V}\left(\bar{\nabla}_{X} Y\right)+g(V, g(X, Y) \xi-\eta(Y) X)-\alpha_{V}\left(\bar{\nabla}_{X} Y\right) \\
& =\left(\nabla_{X} \alpha_{V}\right)(Y)+\eta(V) g(\phi X, \phi Y) \\
& =\left(\nabla_{X} \alpha_{V}\right)(Y)-\eta(V) \alpha_{\phi X}(Y),
\end{aligned}
$$


and hence we obtain the lemma.

For a normal vector field $V \in \Gamma(N L)$, we define the Laplacian of $V$ by

$$
\nabla^{2} V(p)=\sum_{i=1}^{n} \nabla_{\partial_{i}}^{\perp} \nabla_{\partial_{i}}^{\perp} V(p)
$$

at $p \in L$, where $\left(x_{1}, \ldots, x_{n}\right)$ is a geodesic normal coordinate at $p$, and $\partial_{i}=\frac{\partial}{\partial x_{i}}(p), 1 \leq i \leq$ $n$. The Laplacian for 1-forms is defined similarly.

LEMMA 2.2. For $V=f \xi+V_{\mathcal{H}} \in \Gamma(N L)$, we have

$$
\Phi\left(\nabla^{2} V\right)=\left(-\Delta f+2 \delta \alpha_{V}-n f, \nabla^{2} \alpha_{V}+2 d f-\alpha_{V}\right),
$$

where $\Delta=d \delta+\delta d$ is the Laplace-Beltrami operator acting on $C^{\infty}(L)$.

PROOF. By Lemma 2.1, we have

$$
\begin{aligned}
\Phi\left(\nabla^{2} V\right)= & \Phi\left(\sum_{i=1}^{n} \nabla_{\partial_{i}}^{\perp} \nabla_{\partial_{i}}^{\perp} V\right) \\
= & \left(\sum_{i=1}^{n}\left\{\partial_{i}\left(\eta\left(\nabla_{\partial_{i}}^{\perp} V\right)\right)-\alpha_{\nabla_{\partial_{i}}}\left(\partial_{i}\right)\right\}, \sum_{i=1}^{n}\left\{\nabla_{\partial_{i}}\left(\alpha_{\nabla_{\partial_{i}}} V\right)-\eta\left(\nabla_{\partial_{i}}^{\perp} V\right) \alpha_{\phi \partial_{i}}\right\}\right) \\
= & \left(\sum_{i=1}^{n}\left\{\partial_{i}\left(\partial_{i}(\eta(V))-\alpha_{V}\left(\partial_{i}\right)\right)-\left(\nabla_{\partial_{i}} \alpha_{V}-\eta(V) \alpha_{\phi \partial_{i}}\right)\left(\partial_{i}\right)\right\},\right. \\
& \left.\sum_{i=1}^{n}\left\{\nabla_{\partial_{i}}\left(\nabla_{\partial_{i}} \alpha_{V}-\eta(V) \alpha_{\phi \partial_{i}}\right)-\left(\partial_{i}(\eta(V))-\alpha_{V}\left(\partial_{i}\right)\right) \alpha_{\phi \partial_{i}}\right\}\right) \\
= & \left(\sum_{i=1}^{n}\left\{\partial_{i} \partial_{i}(\eta(V))-2 \partial_{i}\left(\alpha_{V}\left(\partial_{i}\right)\right)-\eta(V) d x_{i}\left(\partial_{i}\right)\right\},\right. \\
& \left.\sum_{i=1}^{n}\left\{\nabla_{\partial_{i}} \nabla_{\partial_{i}} \alpha_{V}+2 \partial_{i}(\eta(V)) d x_{i}+\eta(V) \nabla_{\partial_{i}}\left(d x_{i}\right)-\alpha_{V}\left(\partial_{i}\right) d x_{i}\right\}\right) \\
= & \left(-\Delta(\eta(V))+2 \delta \alpha_{V}-n \eta(V), \nabla^{2} \alpha_{V}+2 d(\eta(V))-\alpha_{V}\right),
\end{aligned}
$$

where we put $\alpha_{\phi \partial_{i}}=-d x_{i}(i=1, \ldots, n)$, the dual basis of $\partial_{i}(i=1, \ldots, n)$.

\section{L-minimal Legendrian submanifolds.}

Definition 3.1 ([12]). Let $\left(M^{2 n+1}, \phi, \xi, \eta, g\right)$ be a Sasakian manifold, and $\iota: L^{n} \rightarrow$ $M^{2 n+1}$ a Legendrian immersion. A smooth family $\left\{\iota_{t}\right\}_{-\varepsilon \leq t \leq \varepsilon}$ of immersions of $\iota$ into $M$ is called a Legendrian deformation if $\iota_{t}$ is a Legendrian immersion for each $t$, and $\iota_{0}=\iota$. If $L^{n}$ has boundary, we assume that the deformation $\left\{\iota_{t}\right\}$ fixes the boundary.

Let $V:=\left.\frac{d}{d t}\right|_{t=0} \iota_{t}$ be the variational vector field of $\left\{\iota_{t}\right\}$ along $L$, and denote by $V^{\perp}$ the normal component of $V$. 
PROPOSITION 3.2. A deformation $\left\{\iota_{t}\right\}$ of $L$ is a Legendrian deformation if and only if the variational vector field $V$ satisfies

$$
\left.\alpha_{V^{\perp}}:=-\frac{1}{2} \iota^{*}\left(V^{\perp}\right\rfloor d \eta\right)=\frac{1}{2} d\left(\eta\left(V^{\perp}\right)\right),
$$

or equivalently,

$$
V^{\perp}=f \xi-\frac{1}{2} \phi \nabla f
$$

for some function $f \in C^{\infty}(L)$.

PROOF. By Cartan's formula, we have

$$
\left.\left.\iota^{*}\left(\mathcal{L}_{V} \eta\right)=d(\eta(V))+\iota^{*}(V\rfloor d \eta\right)=d\left(\eta\left(V^{\perp}\right)\right)+\iota^{*}\left(V^{\perp}\right\rfloor d \eta\right)
$$

where $\mathcal{L}$ denotes the Lie derivative. This implies that

$$
\begin{aligned}
\left\{\iota_{t}\right\} \text { is a Legendrian deformation. } & \Leftrightarrow \iota_{t}^{*} \eta=\iota^{*} \eta=0 \text { for each } t \\
& \Leftrightarrow \iota^{*}\left(\mathcal{L}_{V} \eta\right)=0 \\
& \left.\Leftrightarrow d\left(\eta\left(V^{\perp}\right)\right)=-\iota^{*}\left(V^{\perp}\right\rfloor d \eta\right)=2 \alpha_{V^{\perp}} .
\end{aligned}
$$

Moreover, by the isomorphism $\Phi: \Gamma(N L) \rightarrow C^{\infty}(L) \oplus \Omega^{1}(L)$ and (7), we have the identity $V^{\perp}=f \xi-\frac{1}{2} \phi \nabla f$ with $f=\eta\left(V^{\perp}\right)$.

DEFINITION 3.3 ([12]). A variational vector field $V$ is called a Legendrian vector field if it satisfies the condition (9).

REMARK 3.4. Definition 3.1 shows that a Legendrian deformation corresponds to a Lagrangian deformation in the case of Lagrangian submanifolds in Kähler manifolds. However, Proposition 3.2 shows that a Legendrian deformation corresponds to a Hamiltonian deformation, in the sense that $\alpha_{V^{\perp}}$ is an exact 1 -form (see [10]).

Definition 3.5 ([8]). Let $\iota: L^{n} \rightarrow M^{2 n+1}$ be a Legendrian immersion into a Sasakian manifold. Then $\iota$ is called Legendrian minimal (denote L-minimal) if

$$
\left.\frac{d}{d t}\right|_{t=0} \operatorname{Vol}\left(\iota_{t}(L)\right)=0
$$

holds for all Legendrian deformations $\left\{\iota_{t}\right\}$.

The Euler-Lagrange equation under Legendrian deformations is given as follows.

THEOREM 3.6 ([8]). ı is L-minimal if and only if

$$
\delta \alpha_{H}=0, \quad \text { or equivalently, } \operatorname{div} \phi H=0,
$$

where $H$ is the mean curvature vector of $\iota$, and $\delta$ is the codifferential operator on $\Omega^{1}(L)$. 
4. Second variational formula. In this section, we derive the second variational formula under the Legendrian deformation, which leads to the proof of Theorem 1.1.

We start from the general second variational formula. Namely, let $L^{n}$ be an $n$-dimensional compact manifold with or without boundary immersed in a Riemannian manifold $M$ by $\iota: L^{n} \rightarrow M$, and let $\iota_{t}: L^{n} \times(-\varepsilon, \varepsilon) \rightarrow M$ be a deformation of $\iota$ with fixed boundary. Then we have

$$
\begin{aligned}
\left.\frac{d^{2}}{d t^{2}}\right|_{t=0} \operatorname{Vol}\left(\iota_{t}(L)\right) & \\
= & \int_{L}\left\{\sum_{i=1}^{n}\left|\left(\bar{\nabla}_{\partial_{i}} V\right)^{\perp}\right|^{2}+\sum_{i=1}^{n} g\left(\bar{R}\left(\partial_{i}, V\right) \partial_{i}, V\right)-g\left(\bar{\nabla}_{V} V, H\right)\right. \\
& \left.-\sum_{i, j=1}^{n} g\left(\bar{\nabla}_{\partial_{j}} V, \partial_{i}\right) g\left(\bar{\nabla}_{\partial_{i}} V, \partial_{j}\right)+\sum_{i . j=1}^{n} g\left(\bar{\nabla}_{\partial_{i}} V, \partial_{i}\right) g\left(\bar{\nabla}_{\partial_{j}} V, \partial_{j}\right)\right\} d v_{L},
\end{aligned}
$$

where $\left\{\partial_{1}, \ldots, \partial_{n}\right\}$ denotes a geodesic normal frame at a point of $L^{n}, V$ is the variational vector field of $\left\{\iota_{t}\right\}$ at $t=0$, and $H$ is the mean curvature vector of $\iota$. A proof of (10) is given in [14].

Now, we assume the following:

- $\left(M^{2 n+1}, \phi, \xi, \eta, g\right)$ is a Sasakian manifold, and $\iota: L^{n} \rightarrow M^{2 n+1}$ is a compact Legendrian immersion.

- A deformation $\left\{\iota_{t}\right\}$ of $L$ is a Legendrian deformation (i.e., the normal component of $V$ is a Legendrian vector field) with fixed boundary.

- $\iota: L^{n} \rightarrow M^{2 n+1}$ is L-minimal, i.e., $\delta \alpha_{H}=0$.

Since the normal direction of the variational vector field is essential to consider the first and second variations, we may assume that $V$ is normal to $\iota$. We obtain (1) from the general formula (10). The following calculation is due to [14], and seems to be the simplest proof of the second variational formula for Hamiltonian minimal Lagrangian submanifolds in Kähler manifolds.

The first term in (10) is rewritten as

$$
\begin{aligned}
\sum_{i=1}^{n}\left|\left(\bar{\nabla}_{\partial_{i}} V\right)^{\perp}\right|^{2} & =\sum_{i=1}^{n} g\left(\left(\bar{\nabla}_{\partial_{i}} V\right)^{\perp},\left(\bar{\nabla}_{\partial_{i}} V\right)^{\perp}\right) \\
& =\sum_{i=1}^{n}\left\{\partial_{i}\left(g\left(V,\left(\bar{\nabla}_{\partial_{i}} V\right)^{\perp}\right)-g\left(V, \bar{\nabla}_{\partial_{i}}\left(\bar{\nabla}_{\partial_{i}} V\right)^{\perp}\right)\right\}\right. \\
& =-\frac{1}{2} \Delta|V|^{2}-g\left(V, \nabla^{2} V\right)
\end{aligned}
$$

Integrating this, we have from the Gauss theorem,

$$
\text { the first term in }(10)=-\int_{L} g\left(\nabla^{2} V, V\right) d v_{L} .
$$


Next we calculate the fourth and fifth terms in (10). Because

$$
\begin{aligned}
g\left(\bar{\nabla}_{\partial_{i}} V, \partial_{j}\right) & =\partial_{i}\left(g\left(V, \partial_{j}\right)\right)-g\left(V, \bar{\nabla}_{\partial_{i}} \partial_{j}\right) \\
& =-g\left(V,\left(\bar{\nabla}_{\partial_{i}} \partial_{j}\right)^{\perp}\right)\left(\text { since } V \perp \partial_{i}\right) \\
& =-g\left(V, B\left(\partial_{i}, \partial_{j}\right)\right),
\end{aligned}
$$

we have

(12) the fourth and fifth terms in (10) $=-\sum_{i, j=1}^{n} g\left(V, B\left(\partial_{i}, \partial_{j}\right)\right)^{2}+g(V, H)^{2}$.

The third term in (10) is calculated as follows:

LEMma 4.1. Suppose $\iota: L^{n} \rightarrow M^{2 n+1}$ is a compact L-minimal Legendrian immersion into a Sasakian manifold. If $V$ denotes a normal Legendrian vector field along $\iota$, then we have

$$
\int_{L} g\left(\bar{\nabla}_{V} V, H\right) d v_{L}=\int_{L} g(B(\phi V, \phi V), H) d v_{L} .
$$

PROOF. First recall that the (0,3)-tensor $S$ on $L$ defined by

$$
S(X, Y, Z)=g(\phi B(X, Y), Z)
$$

is symmetric over all three variables by the normal condition (see [11], [12]). Hence we obtain

$$
\begin{aligned}
g\left(\bar{\nabla}_{V} V, H\right)-g(B(\phi V, \phi V), H) & =g\left(\phi\left(\bar{\nabla}_{V} V\right), \phi H\right)-g(\phi B(\phi H, \phi V), \phi V) \\
& =g\left(\bar{\nabla}_{V} \phi V, \phi H\right)-g\left(\phi\left(\bar{\nabla}_{\phi H} \phi V\right), \phi V\right) \\
& =\left\{\bar{\nabla}_{V} g(\phi V, \phi H)-g\left(\phi V, \bar{\nabla}_{V} \phi H\right)\right\}+g\left(\bar{\nabla}_{\phi H} V, \phi V\right) \\
& =\bar{\nabla}_{V}\left(\alpha_{V}(\phi H)\right)+\alpha_{V}([\phi H, V]) \\
& =\frac{1}{2}\left\{\bar{\nabla}_{V}(d f(\phi H))+d f([\phi H, V])\right\} \\
& =\frac{1}{2}\{V(\phi H(f))+[\phi H, V](f)\} \\
& =\frac{1}{2} \phi H(V f) \\
& =\frac{1}{2} d(V f)(\phi H),
\end{aligned}
$$

where we use the normal condition and $\alpha_{V}=\frac{1}{2} d f$. Integrating this and using Stokes' theorem, we have

$$
\begin{aligned}
\int_{L}\left\{g\left(\bar{\nabla}_{V} V, H\right)-g(B(\phi V, \phi V), H)\right\} d v_{L} \\
=\frac{1}{2} \int_{L} d(V f)(\phi H) d v_{L} \\
=-\frac{1}{2} \int_{L}\{(V f) \operatorname{div} \phi H\} d v_{L}=-\frac{1}{2} \int_{L}\left\{(V f) \delta \alpha_{H}\right\} d v_{L} .
\end{aligned}
$$


Since we assume $\delta \alpha_{H}=0$, we obtain the lemma.

From (10) through (13), we obtain

$$
\begin{aligned}
\left.(15) \frac{d^{2}}{d t^{2}}\right|_{t=0} \operatorname{Vol}\left(\iota_{t}(L)\right)= & \int_{L}\left\{-g\left(\nabla^{2} V, V\right)+\sum_{i=1}^{n} g\left(\bar{R}\left(\partial_{i}, V\right) \partial_{i}, V\right)-g(B(\phi V, \phi V), H)\right. \\
& \left.-\sum_{i, j=1}^{n} g\left(V, B\left(\partial_{i}, \partial_{j}\right)\right)^{2}+g(V, H)^{2}\right\} d v_{L},
\end{aligned}
$$

and we can prove the next lemma (due to [12, Proposition 4.5]).

LEMMA 4.2. When $\iota: L^{n} \rightarrow M^{2 n+1}$ is a Legendrian immersion into a Sasakian manifold, and $V=f \xi+V_{\mathcal{H}}$ is a normal vector field along $L^{n}$, we have

$$
\begin{aligned}
& \sum_{i=1}^{n} g\left(\bar{R}\left(\partial_{i}, V\right) \partial_{i}, V\right)-\sum_{i, j=1}^{n} g\left(V, B\left(\partial_{i}, \partial_{j}\right)\right)^{2} \\
& \quad=-\overline{\operatorname{Ric}}(V)+g\left(\operatorname{Ric}\left(\alpha_{V}\right), \alpha_{V}\right)+\left|V_{\mathcal{H}}\right|^{2}-g(B(\phi V, \phi V), H)+n f^{2},
\end{aligned}
$$

where $\overline{\operatorname{Ric}}$ is the Ricci curvature of $M$, and Ric is the Ricci transformation acting on $\Omega^{1}(L)$.

PROOF. Since $\left\{\partial_{1}, \ldots, \partial_{n}\right\}$ is an orthonormal basis of $T_{p} L$ at $p \in L^{n},\left\{\phi \partial_{1}, \ldots, \phi \partial_{n}\right\}$ is an orthonormal basis of the horizontal space $\phi\left(T_{p} L\right)$, and thus $\left\{\phi \partial_{1}, \ldots, \phi \partial_{n}, \xi\right\}$ is an orthonormal basis of the normal bundle $N_{p} L$. By the definition of the Ricci curvature, we have

$$
-\overline{\operatorname{Ric}}(V, V)
$$

$$
=\sum_{i=1}^{n}\left\{g\left(\bar{R}\left(\partial_{i}, V\right) \partial_{i}, V\right)\right\}+\sum_{i=1}^{n}\left\{g\left(\bar{R}\left(\phi \partial_{i}, V\right) \phi \partial_{i}, V\right)\right\}+g(\bar{R}(\xi, V) \xi, V) .
$$

Since $V=f \xi+V_{\mathcal{H}}$, the second term in (16) becomes

$$
\begin{aligned}
\sum_{i=1}^{n}\{g & \left.\left(\bar{R}\left(\phi \partial_{i}, V\right) \phi \partial_{i}, V\right)\right\} \\
= & \sum_{i=1}^{n}\left\{g\left(\bar{R}\left(\phi \partial_{i}, V_{\mathcal{H}}\right) \phi \partial_{i}, V_{\mathcal{H}}\right)+2 f g\left(\bar{R}\left(\phi \partial_{i}, \xi\right) \phi \partial_{i}, V_{\mathcal{H}}\right)\right. \\
& \left.+f^{2} g\left(\bar{R}\left(\phi \partial_{i}, \xi\right) \phi \partial_{i}, \xi\right)\right\}
\end{aligned}
$$

where

$$
\begin{aligned}
& \sum_{i=1}^{n} g\left(\bar{R}\left(\phi \partial_{i}, V_{\mathcal{H}}\right) \phi \partial_{i}, V_{\mathcal{H}}\right) \\
& \quad=\sum_{i=1}^{n}\left\{g\left(\bar{R}\left(\partial_{i}, \phi V_{\mathcal{H}}\right) \partial_{i}, \phi V_{\mathcal{H}}\right) \quad\left(\text { since } \phi \partial_{i}, V_{\mathcal{H}} \perp \xi\right)\right.
\end{aligned}
$$




$$
\begin{aligned}
= & \sum_{i=1}^{n}\left\{g\left(R\left(\partial_{i}, \phi V_{\mathcal{H}}\right) \partial_{i}, \phi V_{\mathcal{H}}\right)+g\left(B\left(\partial_{i}, \partial_{i}\right), B\left(\phi V_{\mathcal{H}}, \phi V_{\mathcal{H}}\right)\right)\right. \\
& \left.-g\left(B\left(\phi V_{\mathcal{H}}, \partial_{i}\right), B\left(\partial_{i}, \phi V_{\mathcal{H}}\right)\right)\right\} \text { (by Gauss equation) } \\
= & -\operatorname{Ric}\left(\phi V_{\mathcal{H}}\right)+g\left(H, B\left(\phi V_{\mathcal{H}}, \phi V_{\mathcal{H}}\right)\right)-\sum_{i=1}^{n}\left|B\left(\phi V_{\mathcal{H}}, \partial_{i}\right)\right|^{2} \\
= & -g\left(\operatorname{Ric}\left(\alpha_{V}\right), \alpha_{V}\right)+g\left(H, B\left(\phi V_{\mathcal{H}}, \phi V_{\mathcal{H}}\right)\right)-\sum_{i=1}^{n}\left|B\left(\phi V_{\mathcal{H}}, \partial_{i}\right)\right|^{2} .
\end{aligned}
$$

Moreover, we have

$$
\begin{aligned}
\sum_{i=1}^{n}\left|B\left(\phi V_{\mathcal{H}}, \partial_{i}\right)\right|^{2} & =\sum_{i, j=1}^{n} g\left(B\left(\phi V_{\mathcal{H}}, \partial_{i}\right), \phi \partial_{j}\right)^{2} \\
& =\sum_{i, j=1}^{n} g\left(\phi B\left(\phi V_{\mathcal{H}}, \partial_{i}\right), \partial_{j}\right)^{2} \\
& =\sum_{i, j=1}^{n} g\left(\phi B\left(\partial_{j}, \partial_{i}\right), \phi V_{\mathcal{H}}\right)^{2} \\
& =\sum_{i, j=1}^{n} g\left(B\left(\partial_{j}, \partial_{i}\right), V_{\mathcal{H}}\right)^{2}=\sum_{i, j=1}^{n} g\left(B\left(\partial_{j}, \partial_{i}\right), V\right)^{2},
\end{aligned}
$$

where the third equality follows from the symmetric property of (14). On the other hand,

$$
\begin{aligned}
\bar{R}\left(\phi \partial_{i}, \xi\right) \phi \partial_{i} & =-\left(\bar{\nabla}_{\phi \partial_{i}} \phi\right)\left(\phi \partial_{i}\right) \text { (by the curvature property) } \\
& =-\xi \text { (by the normal condition) }
\end{aligned}
$$

holds, thus the second and the third terms in (17) become

$$
\sum_{i=1}^{n}\left\{2 f g\left(\bar{R}\left(\phi \partial_{i}, \xi\right) \phi \partial_{i}, V_{\mathcal{H}}\right)+f^{2} g\left(\bar{R}\left(\phi \partial_{i}, \xi\right) \phi \partial_{i}, \xi\right)\right\}=-n f^{2} .
$$

Finally, the third term in (16) is rewritten as

$$
\begin{aligned}
g(\bar{R}(\xi, V) \xi, V) & =g(-V+\eta(V) \xi, V) \\
& =-g(V, V)+\eta(V) g(V, \xi) \\
& =-\left(\left|V_{\mathcal{H}}\right|^{2}+f^{2}\right)+f^{2}=-\left|V_{\mathcal{H}}\right|^{2} .
\end{aligned}
$$

Substitute these into (16), we obtain the lemma.

The splitting $V=f \xi+V_{\mathcal{H}}$, into the direction of the characteristic vector field and the horizontal vector field, is a remarkable difference between the case of Legendrian and the case of Lagrangian. In (15), the third, the fourth and the fifth terms in the integrand have essentially no effect on the direction of the characteristic vector field, i.e.,

$$
-g(B(\phi V, \phi V), H)=-g\left(B\left(\phi V_{\mathcal{H}}, \phi V_{\mathcal{H}}\right), H\right),
$$




$$
\begin{aligned}
-\sum_{i, j=1}^{n} g\left(V, B\left(\partial_{i}, \partial_{j}\right)\right) & =-\sum_{i, j=1}^{n} g\left(V_{\mathcal{H}}, B\left(\partial_{i}, \partial_{j}\right)\right) \\
g(V, H)^{2} & =g\left(V_{\mathcal{H}}, H\right)^{2}
\end{aligned}
$$

However, the second term in the right-hand side of (15) can be rewritten similarly as (17), and we have

$$
\sum_{i=1}^{n} g\left(\bar{R}\left(\partial_{i}, V\right) \partial_{i}, V\right)=\sum_{i=1}^{n} g\left(\bar{R}\left(\partial_{i}, V_{\mathcal{H}}\right) \partial_{i}, V_{\mathcal{H}}\right)-n f^{2}
$$

Moreover, by (8), (9) and Lemma 2.2, the first term in the integrand of (15) becomes

$$
\begin{aligned}
-\int_{L} g\left(\nabla^{2} V, V\right) d v_{L} & =-\int_{L}\left\{g\left(\alpha_{\nabla^{2} V}, \alpha_{V}\right)+\eta\left(\nabla^{2} V\right) \eta(V)\right\} d v_{L} \\
& =-\int_{L}\left\{g\left(\nabla^{2} \alpha_{V}+2 d f-\alpha_{V}, \alpha_{V}\right)+\left(-\Delta f+2 \delta \alpha_{V}-n f\right) f\right\} d v_{L} \\
& =\int_{L}\left\{g\left(-\nabla^{2} \alpha_{V}, \alpha_{V}\right)-3 g\left(\alpha_{V}, \alpha_{V}\right)+n f^{2}\right\} d v_{L} .
\end{aligned}
$$

Now, we prove Theorem 1.1.

Proof of TheOrem 1.1. From (15), (18) through (22) and Lemma 4.2, we obtain

$$
\begin{aligned}
& \frac{d^{2}}{\left.d t^{2}\right|_{t=0} \operatorname{Vol}\left(\iota_{t}(L)\right)=} \int_{L}\left[\left\{g\left(-\nabla^{2} \alpha_{V}, \alpha_{V}\right)-3 g\left(\alpha_{V}, \alpha_{V}\right)+n f^{2}\right\}\right. \\
&+\left\{\sum_{i=1}^{n} g\left(\bar{R}\left(\partial_{i}, V_{\mathcal{H}}\right) \partial_{i}, V_{\mathcal{H}}\right)-n f^{2}\right\}-g\left(B\left(\phi V_{\mathcal{H}}, \phi V_{\mathcal{H}}\right), H\right) \\
&\left.-\sum_{i, j=1}^{n} g\left(V_{\mathcal{H}}, B\left(\partial_{i}, \partial_{j}\right)\right)+g\left(V_{\mathcal{H}}, H\right)^{2}\right] d v_{L} \\
&= \int_{L}\left[g\left(-\nabla^{2} \alpha_{V}, \alpha_{V}\right)-3 g\left(\alpha_{V}, \alpha_{V}\right)\right. \\
&+\left\{-\overline{\operatorname{Ric}}\left(V_{\mathcal{H}}\right)+g\left(\operatorname{Ric}\left(\alpha_{V}\right), \alpha_{V}\right)+\left|V_{\mathcal{H}}\right|^{2}-g\left(B\left(\phi V_{\mathcal{H}}, \phi V_{\mathcal{H}}\right), H\right)\right\} \\
&\left.-g\left(B\left(\phi V_{\mathcal{H}}, \phi V_{\mathcal{H}}\right), H\right)+g\left(V_{\mathcal{H}}, H\right)^{2}\right] d v_{L} \\
&= \int_{L}\left\{g\left(-\nabla^{2} \alpha_{V}+\operatorname{Ric}\left(\alpha_{V}\right), \alpha_{V}\right)-2\left|V_{\mathcal{H}}\right|^{2}-\overline{\operatorname{Ric}}\left(V_{\mathcal{H}}\right)\right. \\
&\left.-2 g\left(B\left(\phi V_{\mathcal{H}}, \phi V_{\mathcal{H}}\right), H\right)+g\left(V_{\mathcal{H}}, H\right)^{2}\right\} d v_{L} .
\end{aligned}
$$

It is well known that the Weitzenböck formula implies

$$
-\nabla^{2} \alpha_{V}+\operatorname{Ric}\left(\alpha_{V}\right)=\Delta \alpha_{V},
$$

which leads to (1), since $\alpha_{V}=\frac{1}{2} d f$. 
REMARK 4.3. 1. Oh first proved the second variational formula for Hamiltonian minimal Lagrangian submanifolds in Kähler manifolds under Hamiltonian deformations ([11]). We note that the difference between the second variational formula of L-minimal Legendrian submanifolds and that of H-minimal Lagrangian submanifolds is only the term $-2\left|V_{\mathcal{H}}\right|^{2}$. This term has essentially arisen from the direction of the characteristic vector field of the variational vector field $V$.

2. The second variational formula for the case of minimal Legendrian submanifolds (the case of $H \equiv 0$ ) has been already given in [12]. We generalized his formula in Theorem 1.1 .

Finally, we define the notion of Legendrian stability for L-minimal Legendrian submanifolds:

DEFINITION 4.4. An L-minimal Legendrian submanifold $L^{n}$ in a Sasakian manifold $M^{2 n+1}$ is called Legendrian stable (abbreviated L-stable) if

$$
\left.\frac{d^{2}}{d t^{2}}\right|_{t=0} \operatorname{Vol}\left(\iota_{t}(L)\right) \geq 0
$$

for all Legendrian deformations $\left\{\iota_{t}\right\}$ of $L$.

5. Examples and applications. We apply Theorem 1.1 to investigate the stability of some explicit examples of L-minimal Legendrian submanifolds in Sasakian space forms.

5.1. The stability of $L$-minimal Legendrian curves in Sasakian space forms. Let $\gamma$ be a compact Legendrian curve in a 3-dimensional Sasakian manifold $M^{3}$. In this case, it is easy to prove that the L-minimality condition is equivalent to the condition $|H|=h \equiv$ const (In fact, $h$ is the geodesic curvature of the curve in the standard sense). We also note that all of the Legendrian curves in 3-dimensional Sasakian manifolds have constant torsion equal to 1 ([3]).

By Theorem 1.1, we can easily show that the second variational formula for an Lminimal Legendrian curve in a Sasakian manifold can be rewritten as

$$
\left.\frac{d^{2}}{d t^{2}}\right|_{t=0} \operatorname{Vol}\left(\iota_{t}(\gamma)\right)=\frac{1}{4} \int_{\gamma}\left\{|\Delta f|^{2}-\left(2+h^{2}\right) f \Delta f-\overline{\operatorname{Ric}}(\nabla f)\right\} d v_{\gamma}
$$

since $V_{\mathcal{H}}=-\frac{1}{2} \phi \nabla f$. Moreover, it is easily shown that all 3-dimensional Sasakian manifolds $M^{3}$ are $\eta$-Einstein (i.e., $\left.\overline{\text { Ric }}=a g+(2-a) \eta \otimes \eta\right)$. More precisely, the function $a$ is given by

$$
a(p)=K\left(e_{p}, \phi e_{p}\right)+1, \quad \text { for } p \in M,
$$

where $e_{p}$ is a unit horizontal vector. In this case, $K\left(e_{p}, \phi e_{p}\right)$ is nothing but the sectional curvature of the horizontal subspace $\mathcal{H}_{p}$. Hence, we have from (23),

$$
\left.\frac{d^{2}}{d t^{2}}\right|_{t=0} \operatorname{Vol}\left(\iota_{t}(\gamma)\right)=\frac{1}{4} \int_{\gamma}\left\{|\Delta f|^{2}-\left(a+2+h^{2}\right) f \Delta f\right\} d v_{\gamma},
$$

where the function $a \in C^{\infty}(\gamma)$ is the restriction of (24). 
By (24), we deduce that a 3-dimensional Sasakian manifold is a Sasakian space form with constant $\phi$-sectional curvature $c$ if and only if $a=c+1 \equiv$ const. Hence, for L-minimal Legendrian curves in 3-dimentional Sasakian space forms, we have the following:

COROLlary 5.1. Let $\gamma$ be a compact L-minimal Legendrian curve in a Sasakian space form $M^{3}(c)$. Then $\gamma$ is L-stable if and only if

$$
\lambda_{1} \geq c+3+h^{2},
$$

where $h^{2}=|H|^{2}$ and $\lambda_{1}$ is the first eigenvalue of the Laplace-Beltrami operator $\Delta$ acting on $C^{\infty}(\gamma)$ (If $\gamma$ has boundary, we restrict to smooth functions on $\gamma$ which vanish on the boundary).

Proof. Since $M^{3}(c)$ is a Sasakian space form, we derive from (25) that

$$
\left.\frac{d^{2}}{d t^{2}}\right|_{t=0} \operatorname{Vol}\left(\iota_{t}(\gamma)\right)=\frac{1}{4} \int_{\gamma}\left\{|\Delta f|^{2}-\left(c+3+h^{2}\right) f \Delta f\right\} d v_{\gamma} .
$$

Let $0=\lambda_{0}<\lambda_{1}<\cdots<\lambda_{k}<\cdots \rightarrow \infty$ be the eigenvalues of the Laplace-Beltrami operator $\Delta$ acting on $C^{\infty}(\gamma)$, and $f=f_{0}+\sum_{i=1}^{\infty} f_{i}$ the spectral decomposition of $f \in C^{\infty}(\gamma)$. Then we have

$$
\left.\frac{d^{2}}{d t^{2}}\right|_{t=0} \operatorname{Vol}\left(\iota_{t}(\gamma)\right)=\frac{1}{4} \sum_{i=1}^{\infty} \lambda_{i}\left\{\lambda_{i}-\left(c+3+h^{2}\right)\right\} \int_{\gamma} f_{i}^{2} d v_{\gamma} .
$$

Thus, $\left.\frac{d^{2}}{d t^{2}}\right|_{t=0} \operatorname{Vol}\left(\iota_{t}(\gamma)\right) \geq 0$ holds if and only if $\lambda_{1}-\left(c+3+h^{2}\right) \geq 0$.

REMARK 5.2. If $L^{n}$ has boundary, the above proof should be done with the restriction to functions which vanish on the boundary. Since a Legendrian variational vector field is written as $V=f \xi-\frac{1}{2} \phi \nabla f$, this restriction corresponds to the boundary condition $\left.V\right|_{\partial L}=0$.

EXAMPLE 5.3. The 3-dimensional unit sphere $S^{3}$ is a Sasakian space form with $c=1$ (for more details, see Subsection 5.2 below). It is already known that all the closed L-minimal Legendrian curves in $S^{3}(1)$ are given as

(26) $\gamma(s)=\frac{1}{\sqrt{p+q}}\left(\sqrt{q} e^{\sqrt{-1}(\sqrt{p / q}) s}, \sqrt{-1} \sqrt{p} e^{-\sqrt{-1}(\sqrt{q / p}) s}\right), \quad s \in[0,2 \pi \sqrt{p q}]$,

where $(p, q)$ is a pair of relatively prime positive numbers ([8], [14]). They are torus knots of type $(p, q)$, and in the case of $(p, q)=(1,1)$, it is minimal. It is easy to see that the mean curvature of (26) is given by $h=|H|=|q-p| / \sqrt{p q}$. Since $s$ is the arc length parameter, the first eigenvalue of $\Delta$ of a closed curve is given by $\lambda_{1}=4 \pi^{2} / l^{2}$ where $l$ is the length of the closed curve. Hence, the first eigenvalue of $\Delta$ of (26) is $\lambda_{1}=1 / p q$. Since $p, q$ are positive integers, we have

$$
\lambda_{1}=\frac{1}{p q}<1+3+\frac{(q-p)^{2}}{p q}=\frac{(p+q)^{2}}{p q} .
$$

Thus, by Corollary 5.1, we conclude that all the closed L-minimal Legendrian curves in $S^{3}(1)$ are L-unstable. 
We note that the projection of the curve (26) by the Hopf fibration $\pi: S^{3}(1) \rightarrow \boldsymbol{C} \boldsymbol{P}^{1} \simeq$ $S^{2}$ is a closed curve with constant curvature, and hence a closed circle in $S^{2}$ (i.e., H-minimal Lagrangian closed curve). These curves are all $\mathrm{H}$-stable.

EXAMPLE 5.4. The 3-dimensional Euclidean space $\boldsymbol{R}^{3}$ with the standard Sasakian strucure is a Sasakian space form with constant $\phi$-sectional curvature $c=-3$ (see [5], or Lemma 5.9 below). By Corollary 5.1, minimal Legendrian curves (i.e., $|H|=h=0$, namely, these curves are geodesic) in $\boldsymbol{R}^{3}(-3)$ are all L-stable. In general, all the minimal Legendrian submanifolds in $\boldsymbol{R}^{2 n+1}(-3)$ are L-stable (see [12]). To consider the case $h \neq 0$, we recall that the L-minimality condition implies $h \equiv$ const. A Legendrian curve in $\boldsymbol{R}^{3}(-3)$ with positive constant curvature $h$ is of the form.

$$
\gamma(s)=\mathbf{x}_{0}+\left(\frac{2}{h} \cos h s, \frac{2}{h} \sin h s,-\frac{2}{h} s+\frac{1}{h^{2}} \sin 2 h s+\frac{2 y_{0}}{h} \cos h s\right),
$$

where $\mathbf{x}_{0}=\left(x_{0}, y_{0}, z_{0}\right)$ and $s$ is the arc-length parameter (see [2]). Since Legendrian curves in 3-dimensional Sasakian manifolds have constant torsion, these curves are helices. These curves are not periodic and so non-compact. If we cut the curve with length $l$, namely, if the domain of $s$ is the closed interval $[0, l]$, by Corollary 5.1 , we can easily prove that the above curve is L-stable if and only if $0<h \leq \pi / l$.

EXAMPLE 5.5. Consider the $2 \times 2$ real special linear group $S L(2, \boldsymbol{R})=\{A \in G L(2, \boldsymbol{R})$; $\operatorname{det} A=1\}$. We choose a global coordinate $(x, y, \theta) \in \boldsymbol{R} \times \boldsymbol{R}^{+} \times S^{1}$ of $S L(2, \boldsymbol{R})$ by the unique decomposition

$$
A=\left(\begin{array}{cc}
1 & x \\
0 & 1
\end{array}\right)\left(\begin{array}{cc}
\sqrt{y} & 0 \\
0 & 1 / \sqrt{y}
\end{array}\right)\left(\begin{array}{cc}
\cos \theta & \sin \theta \\
-\sin \theta & \cos \theta
\end{array}\right)
$$

for $A \in S L(2, \boldsymbol{R})$. We define the contact metric structure on $S L(2, \boldsymbol{R})$ by

$$
g=\left(\frac{d x}{2 y}\right)^{2}+\left(\frac{d y}{2 y}\right)^{2}+\left(\frac{d x}{2 y}+d \theta\right)^{2}, \quad \eta=\frac{d x}{2 y}+d \theta, \quad \xi=\frac{\partial}{\partial \theta},
$$

(cf. [4]) and the (1,1)-tensor $\phi$ by the matrix

$$
\phi=\left(\begin{array}{ccc}
0 & 1 & 0 \\
-1 & 0 & 0 \\
0 & -1 / 2 y & 0
\end{array}\right)
$$

Then, $(S L(2, \boldsymbol{R}), \phi, \xi, \eta, g)$ is a Sasakian space form with the $\phi$-sectional curvature $c=-7$. $S L(2, \boldsymbol{R})$ has a compact connected subgroup $S O(2)$, and the homogeneous space $S L(2, \boldsymbol{R})$ / $S O(2)$ is diffeomorphic to the upper half-plane $\boldsymbol{H}^{2}$. By the metric induced from $S L(2, \boldsymbol{R})$, $\boldsymbol{H}^{2}$ has constant Gauss curvature -4 , and the projection $\pi: S L(2, \boldsymbol{R}) \rightarrow \boldsymbol{H}^{2}$ is a Riemannian submersion. Let $\gamma(s)=(x(s), y(s), \theta(s))$ be a smooth curve in $\operatorname{SL}(2, \boldsymbol{R})$ with arc-length parameter. Then $\gamma$ is Legendrian if and only if

$$
\dot{\theta}=-\frac{\dot{x}}{2 y} \text {. }
$$


The Legendrian curve with constant curvature $h$ in $S L(2, \boldsymbol{R})$ is obtained by the horizontal lift of the curve $\bar{\gamma}=(x(s), y(s))$ in $\boldsymbol{H}^{2}$ with constant curvature $h$. If $h>2$, the curve $\bar{\gamma}$ is a closed circle. More precisely, it is given by

$$
(x(s), y(s))=\left(r \sin \mu(s)+x_{0}, r\left(\frac{h}{2}-\cos \mu(s)\right)\right),
$$

where $r \in \boldsymbol{R}^{+}$is a positive constant (cf. [4]), and the smooth function $\mu: \boldsymbol{R} \rightarrow \boldsymbol{R}$ satisfies the equation

$$
\dot{\mu}(s)=h-2 \cos \mu(s)=\frac{2 y(s)}{r},
$$

or more explicitly,

$$
\tan \frac{\mu(s)}{2}=\sqrt{\frac{h-2}{h+2}} \tan \left(\frac{\sqrt{h^{2}-4}}{2} s\right)
$$

with $\mu(0)=0$. Here, we note that

$$
\sin \mu(s)=\frac{\sqrt{h^{2}-4} \sin \left(\sqrt{h^{2}-4} s\right)}{h+2 \cos \left(\sqrt{h^{2}-4} s\right)}, \quad \cos \mu(s)=\frac{2+h \cos \left(\sqrt{h^{2}-4} s\right)}{h+2 \cos \left(\sqrt{h^{2}-4} s\right)} .
$$

By (29) and (30), the Legendrian condition (28) of the horizontal lift $\gamma$ of $\bar{\gamma}$ is rewritten as

$$
\dot{\theta}(s)=-\cos \mu(s)=\frac{\dot{\mu}(s)}{2}-\frac{h}{2} .
$$

Thus $\theta(s)$ is given by

$$
\theta(s)=\frac{\mu(s)}{2}-\frac{h}{2} s
$$

with $\theta(0)=0$. Hence, the horizontal lift of a closed circle in $\boldsymbol{H}^{2}$ with constant curvature $h$ is given by (29) and (33). We note that such a curve is contained in a flat torus in $S L(2, \boldsymbol{R})$ with constant mean curvature $2 h$.

We consider the periodicity of these curves. From (32), $(x(s), y(s))$ is periodic with the period $2 \pi / \sqrt{h^{2}-4}$. Thus the L-minimal Legendrian curve given by (29) and (33) is periodic if and only if there exist a positive integer $m \in \boldsymbol{Z}^{+}$such that, for any $s \in \boldsymbol{R}$,

$$
\theta\left(s+\frac{2 m \pi}{\sqrt{h^{2}-4}}\right) \equiv \theta(s)(\bmod 2 \pi) .
$$

By (33), this is equivalent to

$$
\frac{2 h m \pi}{\sqrt{h^{2}-4}} \equiv \mu\left(s+\frac{2 m \pi}{\sqrt{h^{2}-4}}\right)-\mu(s)(\bmod 2 \pi) .
$$

However, by (31) or (32), the right-hand side of (34) is equal to 0 . Hence, the periodicity condition is equivalent to

$$
h=\frac{2}{\sqrt{1-(m / k)^{2}}}
$$


for some relatively positive integers $m, k \in \boldsymbol{Z}^{+}$with $m / k<1$. Thus there are infinitely many closed L-minimal Legendrian curves in $S L(2, \boldsymbol{R})$ whose curvature is given by (35).

Next, we consider the L-stability of closed L-minimal Legendrian curves in $S L(2, \boldsymbol{R})$. Since the length of a closed L-minimal Legendrian curve given by (29) and (33) with (35) is $l=2 m \pi / \sqrt{h^{2}-4}=\sqrt{k^{2}-m^{2}} \pi$, and since $s$ is an arc-length parameter, the first eigenvalue of $\Delta$ of the curve is $\lambda_{1}=4 \pi^{2} / l^{2}=4 /\left(k^{2}-m^{2}\right)$. Then we have

$$
\lambda_{1}=\frac{4}{k^{2}-m^{2}} \geq-7+3+\frac{4}{1-(m / k)^{2}} .
$$

This inequality is equivalent to $1 \geq m^{2}$. Since $m$ is a positive integer, this inequality holds if and only if $m=1$. Thus, by Corollary 5.1, we conlude that the closed L-minimal Legendrian curve in $S L(2, \boldsymbol{R})$ is L-stable if and only if its curvature is equal to $h=2 / \sqrt{1-(1 / k)^{2}}$ for a positive integer $k>1$.

5.2. Legendrian instability theorem in the unit sphere. In the previous section, we show that every closed L-minimal Legendrian curve in $S^{3}(1)$ is L-unstable. In this section, we generalize this fact, and give a proof of Theorem 1.2.

Let $S^{2 n+1}(1)$ be the unit sphere in the complex Euclidean space $\boldsymbol{C}^{n+1}$, and $i: S^{2 n+1}(1) \hookrightarrow C^{n+1}$ the natural embedding. Since $\boldsymbol{C}^{n+1}$ has the standard Hermitian structure $(J,\langle\rangle$,$) , where J$ is the complex structure and $\langle$,$\rangle is the standard inner product, S^{2 n+1}(1)$ inherits the standard contact metric structure. More precisely, we choose the unit outer normal vector on $S^{2 n+1}(1)$, that is, the position vector $\vec{x} \in S^{2 n+1}(1)$, and we define the characteristic vector field on $S^{2 n+1}(1)$ by $\xi:=-J \vec{x}$. The contact 1 -form on $S^{2 n+1}(1)$ is given by $\eta:=\left\langle i_{*} \cdot, \xi\right\rangle$. The $(1,1)$-tensor $\phi$ on $S^{2 n+1}(1)$ is defined by the relation $J=\phi+\eta \otimes \vec{x}$. We denote by $g$ the induced metric on $S^{2 n+1}(1)$, then $\left(S^{2 n+1}(1), \phi, \xi, \eta, g\right)$ is a contact metric manifold (see [5]). It is well known that this contact metric structure gives a Sasakian structure on $S^{2 n+1}(1)$, and it is a Sasakian space form with constant $\phi$-sectional curvature $c=1$.

Let $\iota: N^{m} \rightarrow S^{2 n+1}(1) \subset \boldsymbol{C}^{n+1} \simeq \boldsymbol{R}^{2 n+2}$ be an immersion of a smooth $m$-dimensional manifold $N^{m}$ into the unit sphere. For a constant vector $a \in \boldsymbol{R}^{2 n+2}$, define the smooth function $\widetilde{f}_{a}$ on $\boldsymbol{R}^{2 n+2}$ by $\widetilde{f}_{a}(x):=\langle a, x\rangle$. Put $\bar{f}_{a}:=i^{*} \widetilde{f}_{a} \in C^{\infty}\left(S^{2 n+1}\right)$ and $f_{a}:=\iota^{*} \bar{f}_{a} \in$ $C^{\infty}(N)$. We note that $\bar{\nabla} \bar{f}_{a}(x)=a-\langle a, x\rangle x$, i.e., the projection of the constant vector $a$ to the tangent space of $S^{2 n+1}(1)$ at $x$.

LEMMA 5.6. We have

$$
\Delta f_{a}=m f_{a}-\langle a, H\rangle,
$$

where $H$ is the mean curvature vector of $l$.

PROOF. Choose the geodesic normal coordinates $\left\{\partial_{1}, \ldots, \partial_{m}\right\}$ at $p \in N$. Then we have

$$
-\Delta f_{a}(p)=\sum_{i=1}^{m}\left(\partial_{i} \partial_{i} f_{a}\right)(p)
$$




$$
\begin{aligned}
& =\sum_{i=1}^{m}\left(\tilde{\nabla}_{\partial_{i}} \tilde{\nabla}_{\partial_{i}}\langle a, \vec{x}\rangle\right)(p) \\
& =\sum_{i=1}^{m}\left(\tilde{\nabla}_{\partial_{i}}\left\langle a, \partial_{i}\right\rangle\right)(p) \quad \text { (since } a \text { is parallel) } \\
& =\sum_{i=1}^{m}\left\langle a, \tilde{\nabla}_{\partial_{i}} \partial_{i}\right\rangle(p) \\
& =\sum_{i=1}^{m}\left\langle a, B\left(\partial_{i}, \partial_{i}\right)-\left\langle\partial_{i}, \partial_{i}\right\rangle \vec{x}\right\rangle(p) \\
& =\left\langle a, H_{p}\right\rangle-m f_{a}(p)
\end{aligned}
$$

REMARK 5.7. If $H \equiv 0$, the above lemma is well known, that is, the function $f_{a}$ is an eigenfunction of the Laplacian $\Delta$ acting on $C^{\infty}(N)$ with the eigenvalue $m=\operatorname{dim} N$.

Proof of Theorem 1.2. Assume that $\iota: L^{n} \rightarrow S^{2 n+1}(1)$ is an L-minimal Legendrian immersion of a compact manifold $L$ without boundary. We show that the immersion $\iota$ is L-unstable. Define the Legendrian vector filed along $\iota$ by $V_{a}:=f_{a} \xi-\frac{1}{2} \phi \nabla f_{a}$, where the function $f_{a}$ is given as above. Since the Ricci tensor of $S^{2 n+1}(1)$ is given by $\overline{\text { Ric }}=2 n g$, the second variational formula becomes

$$
\begin{aligned}
\left.\frac{d^{2}}{d t^{2}}\right|_{t=0} \operatorname{Vol}\left(\iota_{t}(L)\right) \\
\quad=\frac{1}{4} \int_{L}\left\{\left|\Delta f_{a}\right|^{2}-2(n+1) f \Delta f_{a}-2 g\left(B\left(\nabla f_{a}, \nabla f_{a}\right), H\right)+g\left(\phi \nabla f_{a}, H\right)^{2}\right\} d v_{L} .
\end{aligned}
$$

Substituting $\Delta f_{a}=n f_{a}-\langle a, H\rangle$ in this equation, we get

$$
\begin{aligned}
\left.\frac{d^{2}}{d t^{2}}\right|_{t=0} \operatorname{Vol}\left(\iota_{t}(L)\right)=\frac{1}{4} \int_{L}\{ & -n(n+2) f_{a}^{2}+\langle a, H\rangle^{2}+\left\langle\phi \nabla f_{a}, H\right\rangle^{2} \\
& \left.-2\left\langle B\left(\nabla f_{a}, \nabla f_{a}\right), H\right\rangle+2 f_{a}\langle a, H\rangle\right\} d v_{L} .
\end{aligned}
$$

Since $f_{a}=\left.\langle a, \vec{x}\rangle\right|_{L}$, and $\nabla f_{a}=\left(\bar{\nabla} \overline{f_{a}}\right)^{\top}=a^{\top}$ where $\top$ means the projection to the tangent space of $L$, the right-hand side of (37) defines a quadric form on $\boldsymbol{R}^{2 n+2}$. We denote this quadric form on $\boldsymbol{R}^{2 n+2}$ by

$$
\tilde{Q}(a):=\frac{1}{4} \int_{L} Q_{p}(a) d v_{L}, \text { for } a \in \boldsymbol{R}^{2 n+2},
$$

where for each $p \in L$, set

$$
\begin{aligned}
Q_{p}(a):= & -n(n+2) f_{a}^{2}+\langle a, H\rangle^{2}+\left\langle\phi \nabla f_{a}, H\right\rangle^{2} \\
& -2\left\langle B\left(\nabla f_{a}, \nabla f_{a}\right), H\right\rangle+2 f_{a}\langle a, H\rangle .
\end{aligned}
$$

The trace of $Q_{p}$ (and $\tilde{Q}$ ) is independent of the choice of orthonormal basis of $\boldsymbol{R}^{2 n+2}$. Take the orthonormal basis on $\boldsymbol{R}^{2 n+2}$ as $\left\{e_{1}, \ldots, e_{n}, \phi e_{1}, \ldots, \phi e_{n}, \xi, \vec{x}\right\}$, where $x=\iota(p)$ and 
$\left\{e_{1}, \ldots, e_{n}\right\}$ is an orthonormal basis on $\iota_{*}\left(T_{p} L\right)$. Then we have

$$
\begin{aligned}
\operatorname{tr} Q_{p} & =-n(n+2)|\vec{x}|^{4}+\sum_{i=1}^{n}\left\{\left\langle\phi e_{i}, H\right\rangle^{2}+\left\langle\phi e_{i}, H\right\rangle^{2}-2\left\langle B\left(e_{i}, e_{i}\right), H\right\rangle\right\} \\
& =-n(n+2)+2|H|^{2}-2|H|^{2} \\
& =-n(n+2),
\end{aligned}
$$

and hence we have

$$
\operatorname{tr} \tilde{Q}=-\frac{1}{4} n(n+2) \operatorname{Vol}(L)<0 .
$$

This implies that the L-minimal Legendrian immersion $\iota$ is L-unstable.

REMARK 5.8. In [12], a different proof of the L-instability theorem is given under the stronger assumption of compact minimal Legendrian submanifolds in $S^{2 n+1}(1)$. Here we used the averaging method of the second variations due to Lawson-Simons ([9]).

5.3. On the existence of $\mathbf{L}$-minimal Legendrian submanifolds in $\boldsymbol{R}^{2 n+1}(-3)$. The curve (27) is contained in the cylinder in $\boldsymbol{R}^{3}(-3)$ defined by

$$
N^{2}\left(\frac{1}{h}\right):=\left\{(x, y, z) \in \boldsymbol{R}^{3} ;\left(x-x_{0}\right)^{2}+\left(y-y_{0}\right)^{2}=\frac{4}{h^{2}}\right\},
$$

and has constant curvature. By [3, Theorem 4] (see also [2, Theorem 4.2]), such curves are characterized to be of 1-type. However, by [2, Theorem 4.1], an integral submanifold in $\boldsymbol{R}^{2 n+1}(-3)$ is 1 -type if and only if it is minimal in a cylinder. Consequently, a Legendrian curve in $\boldsymbol{R}^{3}(-3)$ lying in a cylinder is L-minimal if and only if it is minimal in a cylinder (we note that all non-minimal L-minimal Legendrian curves in $\boldsymbol{R}^{3}(-3)$ are given by (27), and hence these lie in cylinders). We generalize this situation to Legendrian submanifold in $\boldsymbol{R}^{2 n+1}(-3)$.

Lemma 5.9. Let $L^{n}$ be a Legendrian submanifold in $\boldsymbol{R}^{2 n+1}(-3)$. Assume that $L^{n}$ lies in the cylinder

$$
\begin{aligned}
N^{2 n}(r) & :=\left\{\mathbf{x} \in \boldsymbol{R}^{2 n+1} ; g\left(\mathbf{x}-\mathbf{x}_{\mathbf{0}}, \mathbf{x}-\mathbf{x}_{\mathbf{0}}\right)-\eta\left(\mathbf{x}-\mathbf{x}_{\mathbf{0}}\right)^{2}=r^{2}\right\} \\
& =\left\{\left(x^{1}, \ldots, x^{n}, y^{1}, \ldots, y^{n}, z\right) \in \boldsymbol{R}^{2 n+1} ; \sum_{i=1}^{n}\left(x^{i}-x_{0}^{i}\right)^{2}+\left(y^{i}-y_{0}^{i}\right)^{2}=4 r^{2}\right\},
\end{aligned}
$$

where $\mathbf{x}_{0}:=\left(x_{0}^{1}, \ldots, x_{0}^{n}, y_{0}^{1}, \ldots, y_{0}^{n}, z_{0}\right)$ is a constant vector in $\boldsymbol{R}^{2 n+1}$ and $r$ is constant. Then, for each $p \in L^{n}$, we have

$$
\operatorname{div} \phi H(p)=-\sum_{i=1}^{n} g\left(\bar{\nabla}_{e_{i}} H^{\prime}, \phi e_{i}\right)=-\sum_{i=1}^{n} g\left(\tilde{\nabla}_{e_{i}} H^{\prime}, \phi e_{i}\right),
$$

where $H$ and $H^{\prime}$ are the mean curvature vector of $L^{n}$ in $\boldsymbol{R}^{2 n+1}(-3)$ and in $N^{2 n}(r)$, respectively, $\left\{e_{1}, \ldots, e_{n}\right\}$ is an orthonormal basis of $T_{p} L$, and $\tilde{\nabla}$ is the Levi-Civita connection on the cylinder $N^{2 n}(r)$. 
PROOF. Recall that an odd-dimensional Euclidean space $\boldsymbol{R}^{2 n+1}$ admits the standard Sasakian structure (cf. [2] or [5]), i.e.,

$$
\eta=\frac{1}{2}\left(d z-\sum_{i=1}^{n} y^{i} d x^{i}\right), \quad g=\eta \otimes \eta+\frac{1}{4} \sum_{i=1}^{n}\left(\left(d x^{i}\right)^{2}+\left(d y^{i}\right)^{2}\right), \quad \xi=2 \frac{\partial}{\partial z},
$$

where $\left(x^{1}, \ldots, x^{n}, y^{1}, \ldots, y^{n}, z\right)$ is the standard coordinate of $\boldsymbol{R}^{2 n+1}$, and the (1,1)-tensor $\phi$ is given by the matrix

$$
\phi=\left(\begin{array}{ccc}
0 & \delta_{i j} & 0 \\
-\delta_{i j} & 0 & 0 \\
0 & y^{j} & 0
\end{array}\right)
$$

Then Sasakian manifold $\left(\boldsymbol{R}^{2 n+1}, \phi, \xi, \eta, g\right)$ has constant $\phi$-sectional curvature $c=-3$.

Let $L^{n}$ be a Lengendrian submanifold in $\boldsymbol{R}^{2 n+1}(-3)$ which is contained in a cylinder $N^{2 n}(r)$. Let $H$ be the mean curvature vector of $L^{n}$ in $\boldsymbol{R}^{2 n+1}(-3)$, and let $H^{\prime}$ be the mean curvature vector of $L^{n}$ in $N^{2 n}(r)$. By [2], we have

$$
H_{\mathbf{x}}=H_{\mathbf{x}}^{\prime}+\frac{n}{r^{2}} \phi^{2}\left(\mathbf{x}-\mathbf{x}_{0}\right)
$$

where $\mathbf{x} \in L^{n} \subset \boldsymbol{R}^{2 n+1}$ denotes the position vector of $L^{n}$. We note that $\phi^{2}\left(\mathbf{x}-\mathbf{x}_{0}\right)$ is normal to $N^{2 n}(r)$. Let $\left\{\partial_{1}, \ldots, \partial_{n}\right\}$ be the geodesic normal coordinates of $L^{n}$ at a point of $L^{n}$. Since $\bar{\nabla}_{\partial_{i}}\left(\phi \partial_{i}\right)=\xi$ holds at the point by the normal condition, we have

$$
\begin{aligned}
\operatorname{div} \phi H & =\sum_{i=1}^{n} \partial_{i}\left(g\left(\phi H, \partial_{i}\right)\right)=-\sum_{i=1}^{n} \bar{\nabla}_{\partial_{i}}\left(g\left(H, \phi \partial_{i}\right)\right) \\
& =-\sum_{i=1}^{n}\left\{g\left(\bar{\nabla}_{\partial_{i}} H, \phi \partial_{i}\right)+g(H, \xi)\right\}=-\sum_{i=1}^{n} g\left(\bar{\nabla}_{\partial_{i}} H, \phi \partial_{i}\right)(\text { since } H \perp \xi) \\
& =-\sum_{i=1}^{n}\left\{g\left(\bar{\nabla}_{\partial_{i}} H^{\prime}, \phi \partial_{i}\right)+\frac{n}{r^{2}} g\left(\bar{\nabla}_{\partial_{i}} \phi^{2}\left(\mathbf{x}-\mathbf{x}_{\mathbf{0}}\right), \phi \partial_{i}\right)\right\}
\end{aligned}
$$

where we have

$$
\begin{aligned}
g\left(\bar{\nabla}_{\partial_{i}} \phi^{2}\left(\mathbf{x}-\mathbf{x}_{0}\right), \phi \partial_{i}\right) & =-g\left(\bar{\nabla}_{\partial_{i}}\left(\mathbf{x}-\mathbf{x}_{0}\right), \phi \partial_{i}\right)+g\left(\bar{\nabla}_{\partial_{i}}\left\{\eta\left(\mathbf{x}-\mathbf{x}_{0}\right) \xi\right\}, \phi \partial_{i}\right) \\
& =-g\left(\bar{\nabla}_{\partial_{i}}\left(\mathbf{x}-\mathbf{x}_{0}\right), \phi \partial_{i}\right)-\eta\left(\mathbf{x}-\mathbf{x}_{0}\right) .
\end{aligned}
$$

By [2, Lemma 3.1], since $\partial_{i}$ is tangent to $L^{n}$, we have

$$
\begin{aligned}
& \bar{\nabla}_{\partial_{i}} \mathbf{x}=\partial_{i}-\eta(\mathbf{x}) \phi \partial_{i}+\left(\partial_{i} \eta(\mathbf{x})+g\left(\mathbf{x}, \phi \partial_{i}\right)\right) \xi \\
& \bar{\nabla}_{\partial_{i}} \mathbf{x}_{0}=-\eta\left(\mathbf{x}_{0}\right) \phi \partial_{i}+\left(\partial_{i} \eta\left(\mathbf{x}_{0}\right)+g\left(\mathbf{x}_{0}, \phi \partial_{i}\right)\right) \xi,
\end{aligned}
$$

and hence we obtain

$$
\bar{\nabla}_{\partial_{i}}\left(\mathbf{x}-\mathbf{x}_{0}\right)=\partial_{i}-\eta\left(\mathbf{x}-\mathbf{x}_{0}\right) \phi \partial_{i}+\left(\partial_{i} \eta\left(\mathbf{x}-\mathbf{x}_{\mathbf{0}}\right)+g\left(\mathbf{x}-\mathbf{x}_{\mathbf{0}}, \phi \partial_{i}\right)\right) \xi .
$$

Substituting this into (41), we have

$$
g\left(\bar{\nabla}_{\partial_{i}} \phi^{2}\left(\mathbf{x}-\mathbf{x}_{0}\right), \phi \partial_{i}\right)=0 \text { for } i=1, \ldots, n .
$$


On the other hand, the mean curvature $H^{\prime}$ is a vector contained in the subspace spanned by $\left\{\phi \partial_{1}, \ldots, \phi \partial_{n}\right\}$ in $T \boldsymbol{R}^{2 n+1}(-3)$ because $H^{\prime}$ is normal to $L^{n}$ and $\eta\left(H^{\prime}\right)=\eta(H)=0$. By (44), and since the vector $\phi^{2}\left(\mathbf{x}-\mathbf{x}_{\mathbf{0}}\right)$ is normal to $N^{2 n}(r)$, we have

$$
g\left(\bar{\nabla}_{\partial_{i}} H^{\prime}, \phi^{2}\left(\mathbf{x}-\mathbf{x}_{\mathbf{0}}\right)\right)=\bar{\nabla}_{\partial_{i}} g\left(H^{\prime}, \phi^{2}\left(\mathbf{x}-\mathbf{x}_{\mathbf{0}}\right)\right)-g\left(H^{\prime}, \bar{\nabla}_{\partial_{i}} \phi^{2}\left(\mathbf{x}-\mathbf{x}_{\mathbf{0}}\right)\right)=0 .
$$

This means

$$
\bar{\nabla}_{\partial_{i}} H^{\prime}=\tilde{\nabla}_{\partial_{i}} H^{\prime} \text { for } i=1, \ldots, n,
$$

where $\tilde{\nabla}$ is the Levi-Civita connection on the cylinder $N^{2 n}(r)$.

By (40), (42) and (43), we have

$$
\operatorname{div} \phi H=-\sum_{i=1}^{n} g\left(\bar{\nabla}_{\partial_{i}} H^{\prime}, \phi \partial_{i}\right)=-\sum_{i=1}^{n} g\left(\tilde{\nabla}_{\partial_{i}} H^{\prime}, \phi \partial_{i}\right) .
$$

From Lemma 5.9, we get the following proposition:

PROPOSITION 5.10. Let $L^{n}$ be a Legendrian submanifold in $\boldsymbol{R}^{2 n+1}(-3)$ which lies in a cylinder $N^{2 n}(r)$. If $L^{n}$ has parallel mean curvature in $N^{2 n}(r)$, then $L^{n}$ is L-minimal in $\boldsymbol{R}^{2 n+1}(-3)$.

Proof. Since the mean curvature $H^{\prime}$ is parallel in $N^{2 n}(r)$, i.e., $\tilde{\nabla}^{\perp} H^{\prime}=0, \tilde{\nabla}_{e_{i}} H^{\prime}$ is tangent to $L^{n}$ for each $i=1, \ldots, n$. By Lemma 5.9, we have $\operatorname{div} \phi H=0$, and hence $L^{n}$ is L-minimal.

EXAMPLE 5.11. Define an embedding $\iota: \boldsymbol{R}^{n} \rightarrow \boldsymbol{R}^{2 n+1}(-3)$ by

$$
\begin{aligned}
& \left(s_{1}, \ldots, s_{n}\right) \\
& \mapsto\left(r_{1} \cos \frac{s_{1}}{r_{1}}, \ldots, r_{n} \cos \frac{s_{n}}{r_{n}}, r_{1} \sin \frac{s_{1}}{r_{1}}, \ldots, r_{n} \sin \frac{s_{n}}{r_{n}}, \sum_{i=1}^{n}\left(-\frac{r_{i}}{2} s_{i}+\frac{r_{i}^{2}}{4} \sin \frac{2 s_{i}}{r_{i}}\right)\right),
\end{aligned}
$$

where $r_{1}, \ldots, r_{n}$ are positive constants. This embedding is Legendrian, and lies in a cylinder $N^{2 n}(r)$ with $\mathbf{x}_{\mathbf{0}}=\mathbf{0}, 4 r^{2}=r_{1}{ }^{2}+\cdots+r_{n}{ }^{2}$. This is a generalization of (27), and is the universal covering of the standard torus $T^{n}=S^{1}\left(r_{1}\right) \times \cdots \times S^{1}\left(r_{n}\right)$. By the same calculation as in [2, Example 4.7], the mean curvature vector $H$ is given by

$$
H=-\sum_{i=1}^{n} \frac{4}{r_{i}} \phi\left(\frac{\partial}{\partial s_{i}}\right) .
$$

From this, we can easily show that $\iota$ is L-minimal. By (39), it is easy to check that the mean curvature vector $H^{\prime}$ of $\iota$ in the cylinder is given by

$$
H^{\prime}=\sum_{i=1}^{n}\left(-\frac{4}{r_{i}}+\frac{n}{r^{2}} r_{i}\right) \phi\left(\frac{\partial}{\partial s_{i}}\right) .
$$

Hence, $H^{\prime}$ is parallel in the cylinder if and only if $r_{1}=\cdots=r_{n}$, namely, it is minimal in the cylinder. 
Acknowledgment. The author would like to thank Professors Hiroshi Iriyeh and Jun-ichi Inoguchi for helpful comments on a preliminary version of this paper. He also thanks Professor Reiko Miyaoka for her advice and constant encouragement.

\title{
REFERENCES
}

[1] A. AmARZAYA AND Y. OHNITA, Hamiltonian stability of certain minimal Lagrangian submanifolds in complex projective spaces, Tohoku Math. J. 55 (2003), 583-610.

[2] C. BAIKoussis AND D. E. BLAIR, Finite type integral submanifolds of the contact manifold $\boldsymbol{R}^{2 n+1}(-3)$, Bull. Inst. Math. Acad. Sinica 19 (1991), no. 4, 327-350.

[ 3 ] C. BAikoussis AND D. E. Blair, On Legendre curves in contact 3-manifolds, Geom. Dedicata 49 (1994), 135-142.

[ 4 ] M. Belkhelfa, F. Dillen And J. Inoguchi, Parallel surfaces in the real special linear group $\operatorname{SL}(2, \boldsymbol{R})$, Bull. Austral. Math. Soc. 65 (2002), 183-189.

[ 5 ] D. E. BLAIR, Riemannian geometry of contact and symplectic manifolds, Progress in Math. 203, Birkhäuser, Inc., Boston, MA, 2002.

[6] C. P. Boyer AND K. GALiCKI, Sasakian geometry, Oxford Math. Monogr., Oxford University Press, Oxford, 2008.

[ 7 ] I. CAStro, H. Li AND F. URbano, Hamiltonian-minimal Lagrangian submanifolds in complex space forms, Pacific J. Math. 227 (2006), no. 1, 43-63.

[ 8 ] H. IRIYEH, Hamiltonian minimal Lagrangian cones in $\boldsymbol{C}^{m}$, Tokyo J. Math. 28 (2005), no. 1, 91-107.

[9] H. B. LAWSON, JR. AND J. SimONS, On stable currents and their application to global problems in real and complex geometry, Ann. of Math. 98 (1973), 427-450.

[10] Y. G. ОH, Second variation and stability of minimal Lagrangian submanifolds, Invent. Math. 101 (1990), 501-519.

[11] Y. G. ОH, Volume minimization of Lagrangian submanifolds under Hamiltonian deformations, Math. Zeit. 212 (1993), 175-192.

[12] H. ONO, Second variation and Legendrian stabilities of minimal Legendrian submanifolds in Sasakian manifolds, Differential Geom. Appl. 22 (2005), 327-340.

[13] H. RECKZIEGEL, A correspondence between horizontal submanifolds of Sasakian manifolds and totally real submanifolds of Kähler manifolds, Topics in differential geometry, Vol. I, II (Debrecen, 1984), 1063-1081, Colloq. Math. Soc. János Bolyai, 46, North-Holland, Amsterdam, 1988.

[14] R. Schoen And J. Wolfson, Minimizing area among Lagrangian surfaces: The mapping problem, J. Differential Geom. 58 (2001), 1-86.

\author{
MATHEMATICAL Institute \\ GRADUATE SCHOOL OF SCIENCES \\ TOHOKU UNIVERSITY \\ AOBA-KU, SENDAI, 980-8578 \\ JAPAN \\ E-mail address: sa9m09@math.tohoku.ac.jp
}

